\title{
Modularidad e Integración Morfológica en Cráneos Humanos: un Enfoque Morfométrico Geométrico
}

\author{
Modularity and Morphological Integration in Human Skulls: a Geometric Morphometric Approach
}

\author{
Thomas Püschel*
}

PÜSCHEL, T. Modularidad e integración morfológica en cráneos humanos: un enfoque morfométrico geométrico. Int. J. Morphol., 32(1):299-304, 2014.

RESUMEN: Los conceptos de integración y modularidad refieren al grado de covariación entre los componentes de una estructura. Los módulos morfológicos son aquellas estructuras que poseen componentes que covarían fuertemente, pero que a su vez son relativamente independientes de otros módulos. Mientras que por integración morfológica se entiende a la variación morfológica coordinada de los componentes de un todo funcional. En el caso del cráneo se han definido tradicionalmente dos módulos en base a su origen embrionario diferencial: el esplacnocráneo y el neurocráneo. Se ha sugerido que el desarrollo cráneofacial es altamente integrado, tanto funcional como ontogénicamente. Se puso a prueba la hipótesis de modularidad del desarrollo del cráneo en base a orígenes embrionarios diferenciales, mediante la utilización del coeficiente RV. Posteriormente se evaluó el nivel de integración morfológica entre los módulos definidos anteriormente, mediante un análisis de dos bloques de mínimos cuadrados (PLS). Se intentó conocer así, si la tradicional división entre esplacno y neurocráneo posee un fundamento modular, así como el respectivo nivel de integración morfológica entre ambas estructuras.

PALABRAS CLAVE: Modularidad; Integración Morfológica; Morfometría; Geométrica.

\section{INTRODUCCIÓN}

La modularidad es un importante principio de carácter organizacional en los sistemas biológicos, el cual también se manifestaría a un nivel morfológico (Klingenberg, 2009). Un modulo ha sido definido como una unidad dentro de la cual existe una alta integración de muchas o fuertes interacciones, pero que son relativamente independientes de otras de esas unidades (Klingenberg, 2009). Dependiendo de la problemática de estudio así como del nivel de organización estudiado, pueden variar la naturaleza de la interacciones, las cuales pueden ser del genéticas, del desarrollo y/o funcionales. Desde una perspectiva morfométrica, estas interacciones se manifestarían como una fuerte covariación de los componentes o partes dentro de un modulo, y una débil covariación entre módulos (Klingenberg, 2009). Por su parte la integración refiere al nivel de cohesión entre distintas estructuras, como resultados de las interacciones entre los distintos procesos biológicos que generan el fenotipo en estudio. Usualmente la integración morfológica se infiere a partir del estudio de la covariación entre múltiples rasgos. Por lo tanto, la integración morfológica suele describir el grado en el que una estructu- ra se encuentra ligada a otra debido a diversos procesos (e.g. cascadas del desarrollo en común; funciones biomecánicas coordinadas; pleiotropias) y/o como dos estructuras varían coordinadamente (Klingenberg, 2009). De todas maneras, ambos conceptos refieren al grado de covariación entre los componentes de una unidad: por una parte la modularidad refiere a la relativa independencia de ciertas estructuras, mientras que la integración morfológica concierne a como estas unidades covarían entre sí (Klingenberg, 2009).

El cráneo es una estructura ósea compuesta por la articulación de varios huesos, en cuyo interior se aloja el encéfalo. Es una estructura de alta complejidad e importancia debido a diversas razones. Por una parte participa en diversas funciones, protegiendo a los órganos de los sentidos visión, gusto, audición y olfato, así como al encéfalo, a la vez que soporta a estructuras relacionadas con la masticación, la fonación y la respiración (Lieberman, 2008). Además la morfología cráneofacial es resultado de la expresión génica diferencial, modulaciones epigenéticas y la interacción entre ambos procesos. Desde una perspectiva

* Centre for Anatomical and Human Sciences, Hull York Medical School, University of York, United Kingdom. 
morfológica, esto produce el crecimiento y desarrollo diferencial de los componentes craneales, que da como resultado a su vez una complicada trayectoria ontogénica (Lieberman, 2011). La manera en que como ocurren estos procesos de interacción génico-ambiental, determina en gran manera a la variación morfológica cráneofacial observable. Debido a lo anterior, se ha sugerido que el desarrollo de esta estructura es altamente integrado, tanto funcional como ontogénicamente (Enlow \& Hans, 1998).

La integración morfológica y la modularidad ha sido estudiada en cráneos humanos, definiéndose sus módulos en base principalmente a criterios funcionales y/o orígenes embrionarios diferenciales (Cheverud, 1995; Lieberman $e t$ al., 2002; Bookstein et al., 2003; Ackermann, 2005; Bastir \& Rosas, 2005; Goswami, 2006; Mitteroecker \& Bookstein, 2008). Así usualmente se ha dividido al cráneo en dos grandes regiones en base a su morfogénesis: el esplacnocráneo (viscerocráneo o complejo facial) y el neurocráneo (bóveda craneal o calvaria) (Carlson, 1999). Este último a su vez es sub-divisible en dos regiones adicionales: el basicráneo y el desmocráneo (Sperber, 2001). El primero es osificado endocondralmente a partir de un precursor cartilaginoso (Lieberman, 2008), mientras que el segundo se osificada intramembranosamente a partir de células del mesodermo paraxial y células de la cresta neural (Carlson). No obstante lo anterior, existe un relativo acuerdo de que neurocráneo se desarrolla como un todo funcional impulsado por el crecimiento del encéfalo, mientras que el esplacnocráneo vería mayormente influenciado su desarrollo por otros factores mecánicos tales como la masticación o la fonación (Lieberman, 2011). A pesar del creciente interés relativo a la integración morfológica existente entre las distintas regiones del cráneo, no se ha puesto a prueba la fiabilidad de dichos módulos (Bastir \& Rosas; Goswami; Mitteroecker \& Bookstein).

La presente investigación tuvo por objeto poner a prueba la si la tradicional división entre esplacno y neurocráneo poseía un fundamento modular en cráneos humanos. Posteriormente se dio paso a una evaluación de la integración morfológica entre los distintos módulos definidos anteriormente. En este sentido las preguntas queguiaron esta investigación fueron: 1) ¿Es la tradicional división modular en base a orígenes embrionarios diferenciales entre esplacno y neurocráneo sostenible tras un análisis modular? y 2) ¿Cuál es el nivel de integración morfológica entre esplacno y neurocráneo?

\section{MATERIAL Y MÉTODO}

Muestra. Se utilizaron los registros fotográficos laterales izquierdos de 116 cráneos arqueológicos provenientes del Norte de Chile. Estos documentos fotográficos se encuentran resguardados en el Laboratorio de Morfometría Geométrica, Programa de Genética Humana, ICBM, Universidad de Chile.

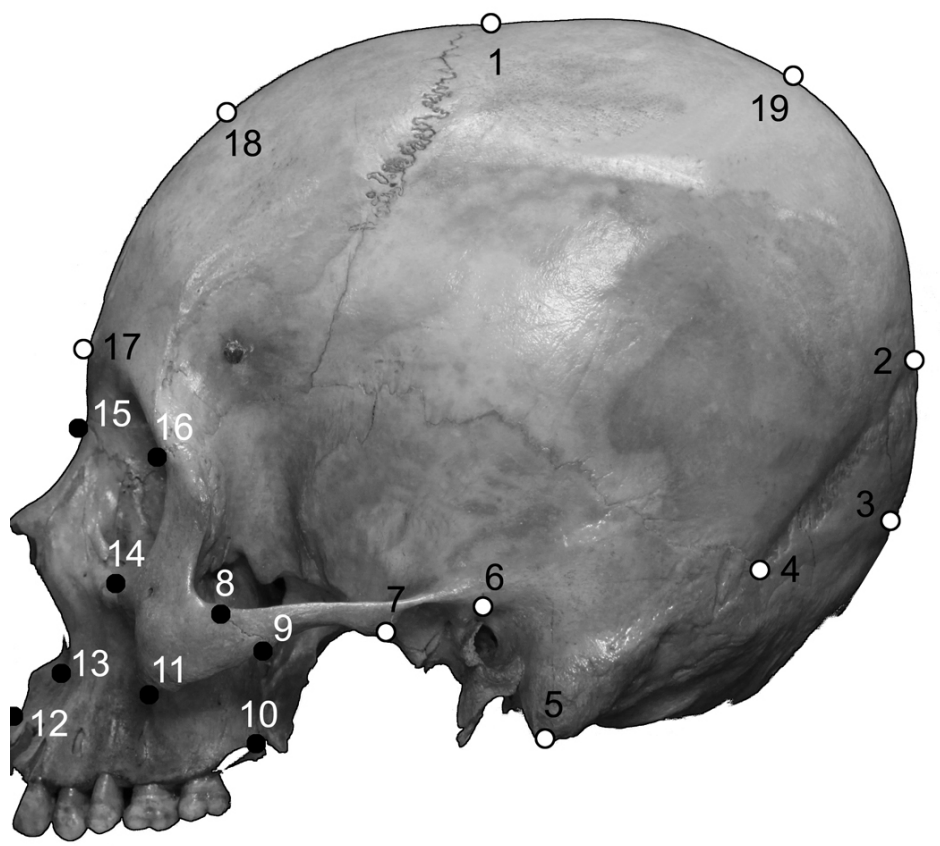

Fig.1. Mapa de Hitos Homólogos utilizados. Los hitos negros representan al esplacnocráneo, mientras que los blancos representan al neurocráneo.
Los criterios de inclusión para las fotografías fueron:

a) Muestras de cráneos de individuos de ambos sexos y adultos, con cierre evidente de la sincondrosis esfenooccipital y/o erupción del tercer molar definitivo.

b) Muestras que presenten una conservación suficiente de las relaciones morfológicas cráneofaciales.

c) Muestras que presenten una integridad adecuada de las estructuras morfológicas que definen los hitos anatómicos homólogos en la radiografías con norma lateral.

Los criterios de exclusión de la muestra fueron:

a) Aquellas muestras que contradigan alguno de los criterios de inclusión, tales como: individuos infantiles, de sexo desconocido, con solución de continuidad en alguna de sus estructuras anatómicas, o fragmentados. 
b) Fotografías de escasa nitidez para distinguir hitos homólogos o imágenes radiográficas con evidentes distorsiones por ampliación o deformación.

c) Individuos que presenten signos de haber sido deformados artificialmente.

Obtención del dato primario. Se tomaron fotografías en vista lateral utilizando una cámara fotográfica Canon SLR digital EOS Rebel XS®. La cámara fue montada sobre un trípode a 1.5 metros de distancia del cráneo. Este último fue posicionado de acuerdo al plano de Frankfurt, mediante la utilización de niveles. El cráneo se orientó lateralmente de acuerdo a la sutura sagital y frontalmente de acuerdo al punto craneométrico de Porion. Junto al cráneo y alineada también, se colocó una escala metálica de 15 centímetros. Para no tener distorsiones o deformaciones asociadas a problemas de alineamiento de los especímenes, se siguieron todas las recomendaciones descritas en Zelditch et al. (2004).

Procesamiento del dato primario. Los datos morfométricos fueron colectados como coordenadas 2D de hitos anatómicos homólogos mediante la utilización del programa tpsDig v. 2.16 (Rohlf, 2001). En cada cráneo fueron digitalizados 19 hitos anatómicos (Fig. 1).

Se eliminaron las diferencias debidas a rotación, traslación y escala a través de un análisis de Procusto generalizado (GPA). Como estimador del tamaño se utilizó el tamaño del centroide (Bookstein et al.). Los análisis morfométrico geométricos y estadísticos se llevaron a cabo utilizando el programa MorphoJ (Klingenberg, 2011).

Coeficiente RV. Con el fin de la evaluar una hipótesis de modularidad se especificó la partición de la configuración de hitos homólogos en dos sub-conjuntos que correspondieron a los módulos hipotéticos (esplacnocráneo y neurocráneo). Posteriormente se comparó la fuerza de la covariación entre los módulos contra un número de posibles particiones alternativas con igual número de hitos homólogos que los módulos hipotéticos, mediante el cálculo del coeficiente RV (Klingenberg, 2009). Este coeficiente puede ser considerado un análogo multivariado de una correlación (Escoufier, 1973), y fue calculado entre los dos módulos hipotéticos, y entre el conjunto de particiones alternativas generando una distribución de valores.

PLS. El método de mínimos cuadrados parciales (PLS) se utiliza para estudiar los patrones de covariación entre dos o más conjuntos de variables. Su utilidad en morfometría geométrica radica en que al menos unos de estos dos o más conjuntos de variables contienen componentes de la forma (Klingenberg, 2011). Por tanto se puede usar al PLS para relacionar a dichos componentes de la forma con otros tipos de datos tales como condiciones experimentales, información ecológica u otras variables de la forma. El análisis de PLS de dos bloques se basa en el método de descomposición de valor único (SVD) de la matriz de covarianza entre los dos conjuntos de variables (Rohlf \& Corti, 2000; Zelditch et al.). Básicamente este análisis encontrar pares correlacionados de combinaciones lineales (vectores singulares) entre dos conjuntos (o bloques) de variables, que den cuenta de la mayor covariación posible entre los dos bloques de variables originales (Rohlf \& Corti). El objetivo es intentar maximizar la representación en pocas dimensiones de la estructura de covariancia entre los conjuntos de variables (o bloques) (Zelditch et al.).

\section{RESULTADOS}

Hipótesis de Modularidad. Primero se analizó la modularidad del cráneo humano proponiendo dos módulos del desarrollo (esplacno y neurocráneo), utilizando el coeficiente RV. El resultado aquí presentado muestra una perfecta correspondencia los módulos definidos de antemano y aquellos definidos como las particiones alternativas de menor covariación, lo cual es una fuerte evidencia para apoyar la división modular basada en orígenes del desarrollo diferenciales (neurocráneo y esplacnocráneo). La distribución de los coeficientes RV muestra que la hipótesis a priori se encuentra en el extremo izquierdo de la curva de distribución, siendo la partición con el coeficiente $\mathrm{RV}$ más bajo $(\mathrm{RV}=$ 0,280958) (Fig. 2.). De un total de 6491 particiones alternativas a las que se les calculó el coeficiente RV ninguna presenta un coeficiente RV menor que el hipotetizado de antemano. El análisis aquí presentado es una buena evidencia para sostener la organización modular por orígenes del desarrollo diferenciales.

Integración Morfológica. Tras comprobar que la cara ósea y la bóveda craneal se comportan modularmente se procedió a estudiar la covariación entre ambos módulos para así calcular el nivel de integración morfológica entre los módulos. El coeficiente RV aquí se utilizó como una medida general para la fuerza de la asociación entre los bloques. Como se explicó previamente, este coeficiente puede ser interpretado como un análogo multivariado de un coeficiente de correlación entre dos variables (Escoufier). Esto significa que sólo hay una leve aunque significativa integración morfológica entre neurocráneo y cara $(\mathrm{RV}=0,282923$; $\mathrm{p}$ valor $(1000$ rondas de permutación $)=<0,001)$. Los resultados del PLS para los cráneos reflejan el mismo resultado (Tabla I). Los resultados fueron significativos tanto para los primeros ocho valores singulares ( $\mathrm{p}$-valor $<0,001)$ y para varias correlaciones pareadas de los valores PLS $(p<0,05)$ 


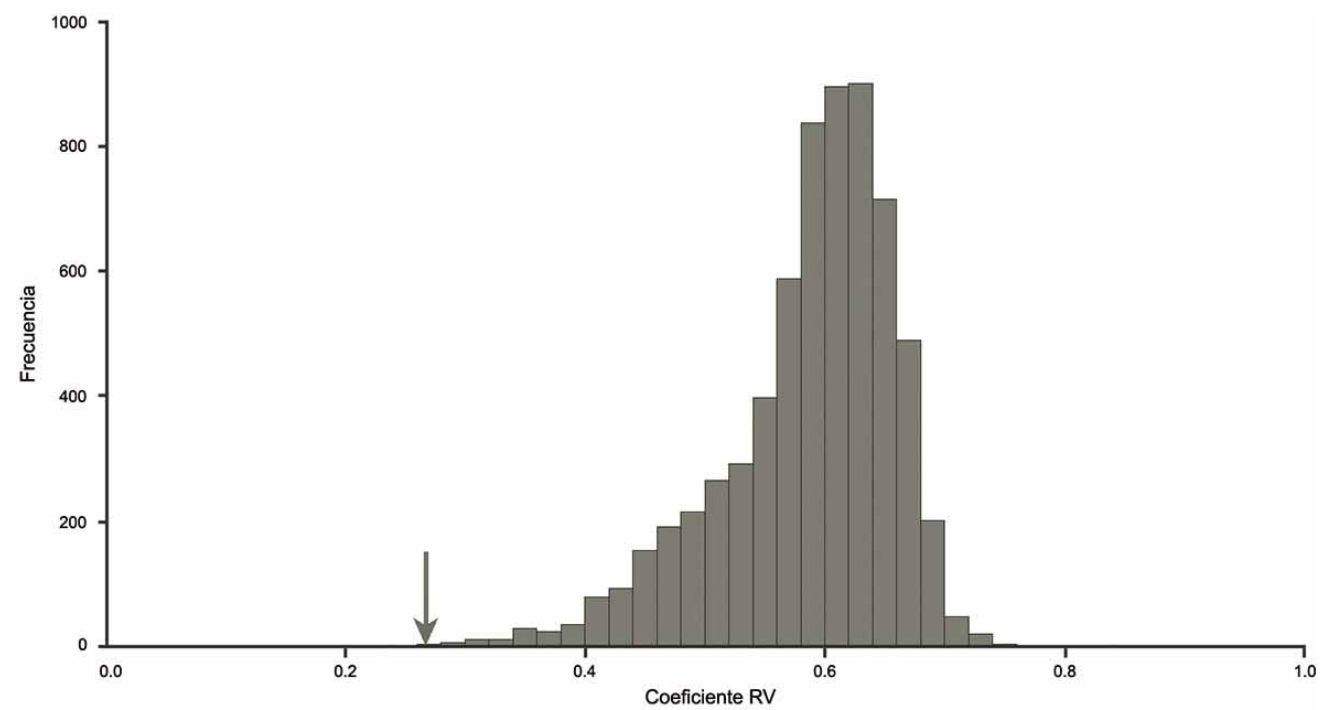

Fig. 2. Histograma de la distribución de los 6491 coeficientes RV calculados a partir de módulos generados aleatoriamente y con igual número de hitos homólogos que los módulos hipotetizados a priori. La flecha indica el valor del coeficiente RV calculado para los módulos propuestos. La hipótesis a priori se encuentra en el extremo izquierdo de la curva de distribución, siendo la partición con el coeficiente RV más bajo $(\mathrm{RV}=0,280958)$.

Tabla I. Valores singulares y correlaciones pareadas para los puntajes PLS entre Esplacno y Neurocráneo.

\begin{tabular}{lccccc}
\hline & Valor Singular & Valor P $($ perm.) & \% Covariación Total & Correlación & Valor P (perm.) \\
\hline PLS1 & 0,00020812 & $<0,001$ & 35,454 & 0,58356 & 0,032 \\
PLS2 & 0,00020355 & $<0,001$ & 33,914 & 0,83862 & $<0,001$ \\
PLS3 & 0,0001301 & $<0,001$ & 13,855 & 0,63201 & 0,009 \\
PLS4 & 0,0001012 & $<0,001$ & 8,382 & 0,74689 & $<0,001$ \\
PLS5 & 0,00008189 & $<0,001$ & 5,490 & 0,59366 & $<0,001$ \\
PLS6 & 0,00003964 & $<0,001$ & 1,286 & 0,44866 & 0,012 \\
PLS7 & 0,0000293 & 0,001 & 0,703 & 0,47252 & 0,001 \\
PLS8 & 0,00002184 & 0,013 & 0,391 & 0,50931 & $<0,001$ \\
PLS9 & 0,00001514 & 0,226 & 0,188 & 0,34574 & 0,047 \\
PLS10 & 0,00001321 & 0,092 & 0,143 & 0,2739 & 0,282 \\
PLS11 & 0,00001116 & 0,049 & 0,102 & 0,35532 & 0,001 \\
PLS12 & 0,00000636 & 0,854 & 0,033 & 0,24351 & 0,163 \\
PLS13 & 0,00000513 & 0,776 & 0,022 & 0,22514 & 0,15 \\
PLS14 & 0,00000484 & 0,233 & 0,019 & 0,19426 & 0,155 \\
PLS15 & 0,00000376 & 0,175 & 0,012 & 0,20467 & 0,023 \\
PLS16 & 0,0000023 & 0,429 & 0,004 & 0,15561 & 0,057 \\
PLS17 & 0,00000154 & 0,385 & 0,002 & 0,20495 & $<0,001$ \\
PLS18 & 0,00000097 & 0,157 & 0,001 & 0,08085 & 0,053 \\
\hline
\end{tabular}

Valores significativos en negrita $(\mathrm{a}=0,05)$, tras 1000 rondas de permutación.

(Tabla I). Los primeros dos pares de ejes singulares dieron cuenta del $36,249 \%$ de la covarianza total de la muestra, mientras que los segundos dos pares explicaron el $31,232 \%$. La Figura 3 muestra el patrón de covariación del primer par de ejes singulares para los 116 individuos analizados, evidenciándose visualmente la covariación entre ambos. Interesantemente la covariación entre los módulos para el
PLS1 puede ser descrita como una expansión en la porción superior del neurocráneo (huesos parietales y frontal) y una proyección hacia anterior de la porción basilar del occipital. Por su parte la porción superior del esplacnocráneo (orbitas y cigomáticos) muestran una retracción, mientras que la porción inferior (procesos alveolares) presentan una proyección antero-inferior. 
Fig. 3. Gráfico de los puntajes PLS de los 116 individuos analizados para el primer par de ejes singulares para los dos módulos: esplacnocráneo y neurocráneo.

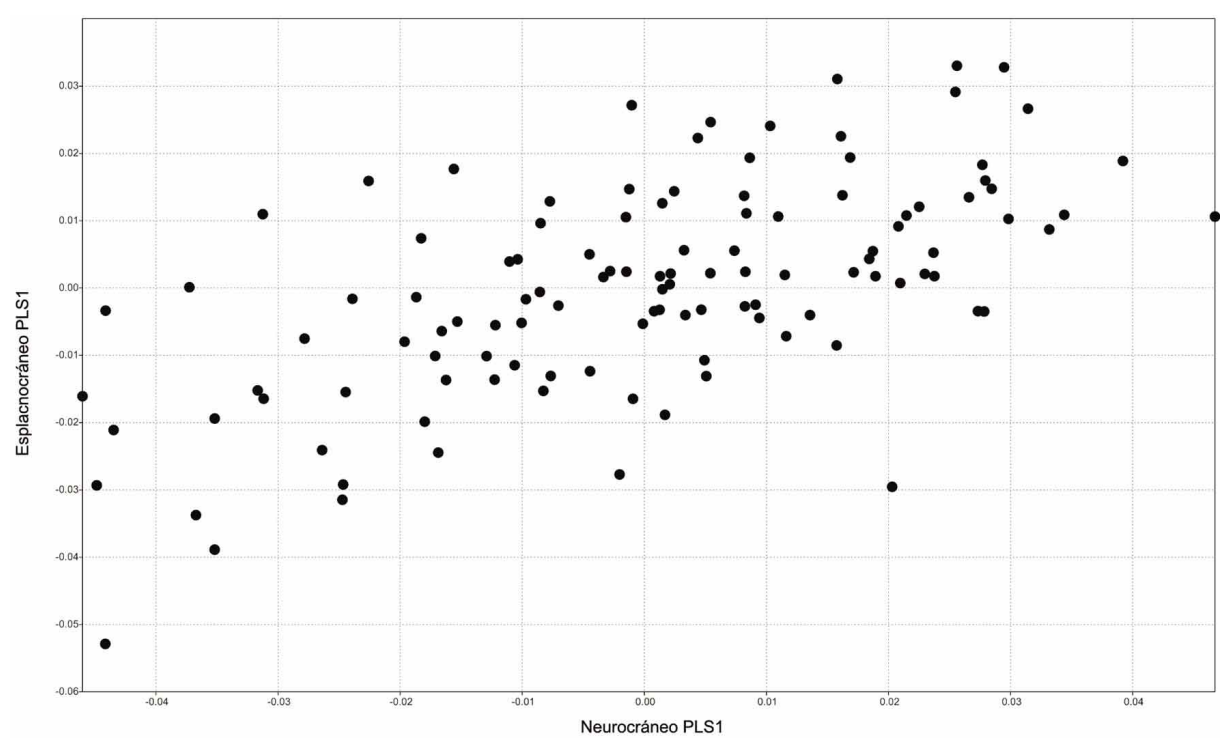

\section{DISCUSIÓN}

En base a la evidencia aquí presentada, es factible sostener que los componentes del cráneo humano se encuentran integrados entre ellos. Los resultados del análisis de PLS muestra que los primeros ocho pares de ejes singulares que explicaron la mayor parte de la covariación total de la muestra fueron significativos $(98,38 \%)$, indicando que existe una integración entre distintas porciones del cráneo con orígenes embrionarios diferenciales. Aunque la causa próxima de los patrones de covariación observados es aún desconocida, esta integración morfológica probablemente surge debido a que los componentes del cráneo evolucionan, se desarrollan y funcionan de manera conjunta y coordinada (Lieberman, 2011). La integración morfológica de las partes del cráneo es por tanto inevitable, debido a que estos componentes comparten precursores del desarrollo, se ubican espacialmente cercanos, diferentes funciones los afectan conjuntamente y porque comparten una historia evolutiva común (Lieberman, 2008). Futuros trabajos acerca de este tópico, podrían intentar dilucidar las posibles causas que subyacen a cada uno de estos patrones de covariación en especifico.

No obstante el fenómeno de integración morfológica entre esplacnocráneo y neurocráneo haya sido puesto a prueba positivamente, de igual manera se evidenció que esta covariación no es total, sino que estructurada modularmente y relativamente leve. El presente trabajo pudo testear cuantitativamente la hipótesis de modularidad propuesta en base a orígenes embrionarios diferenciales, entre esplacnocráneo y neurocráneo. De esta manera se pudo evidenciar, que ambos módulos existen y que se comportan de manera relativamente independiente. Investigaciones futu- ras con respecto a este tema, podrían intentar dilucidar la secuencia ontogénica seguida durante la conformación de estos módulos.

Finalmente, se mostró mediante un ejemplo concreto, lo adecuado que resulta la aplicación de la morfometría geométrica para estudiar los fenómenos de modularidad e integración morfológica. Estos métodos ofrecen una alta flexibilidad y potencia estadística, junto con unos bien desarrollados y coherentes fundamentos matemáticos (Klingenberg, 2008). La combinación entre geometría y métodos estadísticos multivariados, aseguraron una caracterización completa y no redundante de las formas bajo estudio, y por tanto una adecuada representación de las morfologías de interés. Permitiendo de esta manera, el análisis cuantitativo y multivariado de los fenómenos de integración morfológica y modularidad en cráneos humanos.

\section{AGRADECIMIENTOS}

Agradezco a Philippe Menecier (Musée de l'Homme, Paris), Bernardo Arriaza (Museo Arqueológico San Miguel de Azapa, Arica) y Manuel Arturo Torres (Museo G. Le Paige, San Pedro de Atacama) por el acceso a los especímenes bajo su cuidado. También agradezco a Germán Manríquez por las interesantes discusiones acerca del tópico aquí tratado y por el apoyo a esta investigación. Este trabajo ha sido financiado parcialmente por el Programa de Investigación Asociativa Anillos en Ciencia y Tecnología ACT No 096 y por Becas Chile, Conicyt-PCHA/2012/73130010. 
PÜSCHEL T. Modularity and morphological integration in human skulls: a geometric morphometric approach. Int. J. Morphol., 32(1):299304, 2014.

SUMMARY: Morphological Integration and modularity are concepts that refer to the covariation level between the components of a structure. Morphological modules are those structures that have components that strongly covary, which in turn are relatively independent to other modules. Morphological integration is instead, the coordinated morphological variation of a functional whole. Traditionally the skull has been divided in two modules based on their different developmental origins: the splanchnocranium and the neurocranium. It has been suggested that the craniofacial development is highly integrated both functional as ontogenetically. The modularity hypothesis based on different developmental origins was tested, by using the RV coefficient. Later, the integration level was assessed applying a partial least-squares analysis (PLS). The underlying aim was to know whether the traditional division between splanchno and neurocranium has a modular basis, as well as the morphological integration level between these two structures.

KEY WORDS: Modularity; Morphological Integration; Geometric Morphometrics.

\section{REFERENCIAS BIBLIOGRÁFICAS}

Ackermann, R. R. Ontogenetic integration in the hominoid face. $J$. Hum. Evol., 48(2):175-97, 2005.

Bastir, M. \& Rosas A. Hierarchical nature of morphological integration and modularity in the human posterior face. Am. J. Phys. Anthropol., 128(1):26-34, 2005.

Bookstein, F. L.; Gunz P.; Mitteroecker P.; Prossinger H.; Schaefer K. \&. Seidler, H. Cranial integration in Homo: singular warps analysis of the midsagittal plane in ontogeny and evolution. $J$. Hum. Evol., 44(2):167-87, 2003.

Carlson, B. Human Embryology And Developmental Biology. Philadelphia, Mosby Elsevier, 1999.

Cheverud, J. M. Morphological integration in the saddle-back tamarin (Saguinus fuscicollis) cranium. Am. Nat., 145(1):63-89, 1995.

Enlow, D. \& Hans, M. Crecimiento Facial. México D.F., McGrawHill Interamericana, 1998.

Escoufier, Y. Le traitement des variables vectorielles. Biometrics, 29(4):751-60, 1973.

Goswami, A. Cranial modularity shifts during mammalian evolution. Am. Nat., 168(2):270-80, 2006.

Klingenberg, C. P. Morphological integration and developmental modularity. Annu. Rev. Ecol. Evol. Syst., 39:115-32, 2008.

Klingenberg, C. P. Morphometric integration and modularity in configurations of landmarks: Tools for evaluating a-priori hypotheses. Evol. Dev., 11(4):405-21, 2009.

Klingenberg C. P. MorphoJ: an integrated software package for geometric morphometrics. Mol. Ecol. Resour., 11(2):353-7, 2011.

Lieberman, D. E.; McBratney, B. M. \& Krovitz, G. E. The evolution and development of cranial formin Homo sapiens. Proc. Natl. Acad. Sci. U. S. A., 99(3):1134-9, 2002.
Lieberman, D. E. Speculations about the selective basis for modern human craniofacial form. Evol. Anthropol., 17:55-68, 2008.

Lieberman, D. E. Evolution of the Human Head. Massachussets, Harvard University Press, 2011.

Mitteroecker, P. \& Bookstein, F. The Evolutionary Role of Modularity and Integration in the Hominoid Cranium. Evolution, 62(4):943$58,2008$.

Rohlf, F. J. \& Corti, M. The use of two-block partial least-squares to study covariation in shape. Syst. Biol., 49(4):740-53, 2000.

Rohlf, F. J. TPSdig, v. 1.31. New York, State University at Stony Brook, 2001.

Sperber, G. H. Craniofacial Development. London, BC Decker Inc., 2001.

Zelditch, M. L.; Swiderski, H. D.; Sheets D. H. \& Fink, W. L. Geometric Morphometrics for Biologists: A primer. New York, Elsevier Academic Press, 2004.

Dirección para Correspondencia:

Thomas Püschel

Centre for Human and Anatomical Sciences

Hull York Medical School

University of York

Heslington, York Y010 5DD

UNITED KINGDOM

Email: thomaspuschel@gmail.com

Recibido: 25-08-2013

Aceptado: 16-12-2013 